\title{
On type I planetary migration in adiabatic disks
}

\author{
Clément Baruteau ${ }^{1}$ and Frédéric Masset ${ }^{1,2}$ \\ ${ }^{1}$ Laboratoire AIM, CEA/DSM - CNRS - Université Paris Diderot \\ DAPNIA/Service d'Astrophysique, CEA/Saclay, 91191 Gif/Yvette Cedex, France \\ email: clement.baruteau@cea.fr \\ ${ }^{2}$ IA-UNAM, Ciudad Universitaria, Apartado Postal 70-264, Mexico D.F. 04510, Mexico \\ email: fmasset@cea.fr
}

\begin{abstract}
We investigate type I migration in a two-dimensional adiabatic disk. We find entropy perturbations that are advected in the planet's coorbital region. These entropy perturbations yield an excess of corotation torque that scales with the unperturbed entropy gradient at corotation. This torque excess can be large enough to slow down migration significantly, or even stop it.
\end{abstract}

Keywords. accretion, accretion disks, hydrodynamics, methods: numerical

\section{Introduction}

Recently, Paardekooper \& Mellema (2006) have revisited type I migration with highresolution $3 \mathrm{D}$ calculations, including radiative transfer. They find that the migration can be reversed for sufficiently large values of the disk opacity, which may solve the lingering problem of type I migration. This work is a first step to further investigate this topic. For this purpose, we consider a more restricted situation, namely $2 \mathrm{D}$ adiabatic flows.

\section{Adiabatic vs. isothermal calculations}

We performed 2D calculations of a low-mass planet embedded in an inviscid gaseous disk. In its unperturbed state, the disk has axisymmetric surface density and temperature profiles, which read, respectively, $\Sigma(r) \propto r^{-0.5}$ and $T(r) \propto r^{-0.6}$. The gas pressure $p$ is given by $p=\Sigma T$. The disk is perturbed by a planet on a fixed circular orbit. The planet to primary mass ratio is $q=2.2 \times 10^{-5}$, and the softening length of the planet potential is $\varepsilon=0.03 r_{p}$, where $r_{p}$ denotes the planet's orbital radius.

Two series of runs were performed:

- The disk is described by a locally isothermal equation of state, as is customarily assumed in disk-planet calculations.

- The disk is described by an adiabatic energy equation. This equation corresponds to the Lagrangian conservation of the gas entropy, referred to as the quantity $S=p / \Sigma^{\gamma}$, where $\gamma$ is the adiabatic index $(\gamma=1.4$ in our calculations).

Figure 1 shows the relative perturbation of the gas surface density in both situations. The planet's wake is less tightly wound in the adiabatic case, since the adiabatic sound speed $c_{s}$ is larger than the isothermal one, by a factor of $\sqrt{\gamma}$. The differential Lindblad torque, which scales as $c_{s}^{-2}$ (Ward 1997), is therefore reduced by a factor of $\gamma$ in an adiabatic disk. The same is true of the corotation torque, when there is no entropy gradient (Baruteau \& Masset 2008). Furthermore, the adiabatic calculation displays additional 

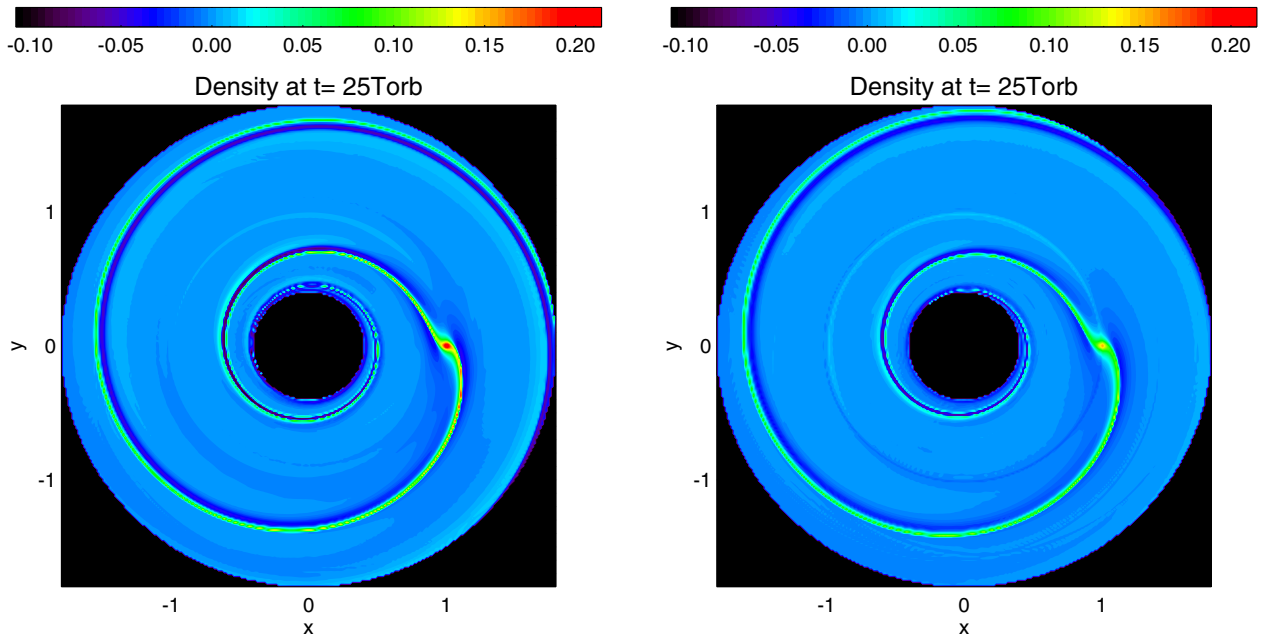

Figure 1. Relative perturbation of the gas surface density obtained with a locally isothermal equation of state (left panel), and with an adiabatic energy equation (right panel). The planet is located at $x=1, y=0$.

density perturbations, located in the planet's coorbital region. These perturbations can have a dramatic impact on the corotation torque, as we shall see.

\section{Additional density perturbations in the planet's coorbital region}
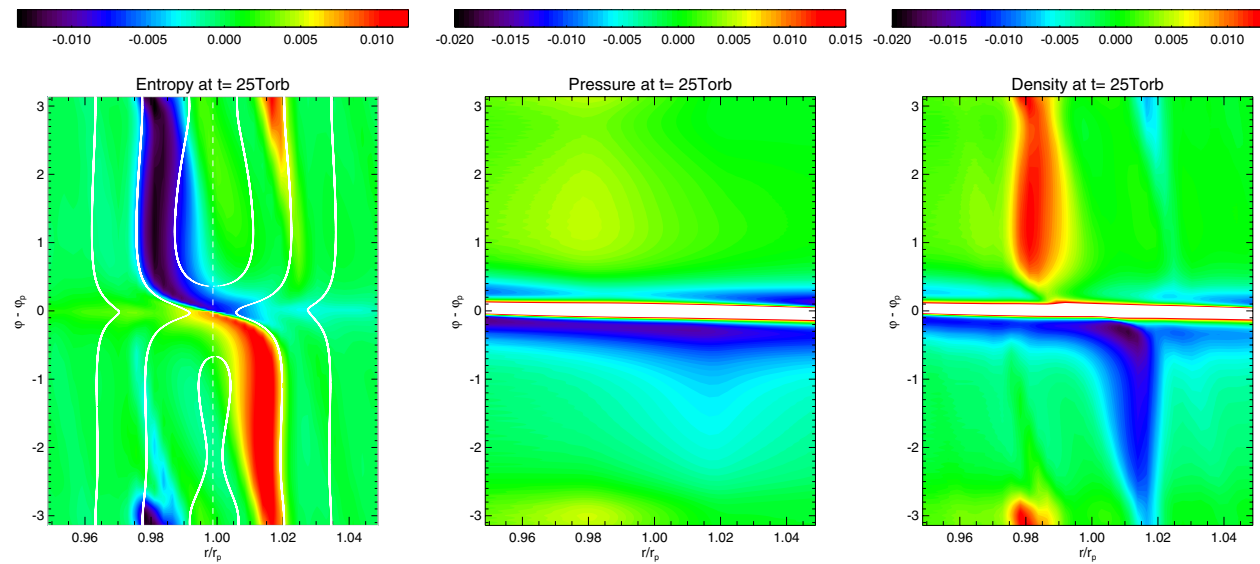

Figure 2. Relative perturbations of the gas entropy, pressure and surface density obtained with the adiabatic calculation of section 2 . The planet is located at $r=r_{p}, \varphi=\varphi_{p}$. In the entropy panel, the vertical dashed line represents the planet's corotation radius, and streamlines are overplotted to show the extent of the planet's coorbital region. In the pressure and surface density panels, the nearly horizontal (saturated) structure at $\varphi=\varphi_{p}$ is the planet's wake. The planet's Hill sphere radius is $R_{H} \approx 0.02 r_{p}$.

We display in figure 2 the relative perturbations of the gas entropy, pressure and surface density obtained with the adiabatic calculation of section 2 . While the azimuthal range spans the whole $[0,2 \pi]$ interval, the radial range depicted is restricted to a band of width $2.5 x_{s}$ around the corotation radius, where $x_{s}$ denotes the half-width of the 
planet's coorbital region. Streamlines are overplotted to the entropy panel to show the extent of the coorbital region, and the vertical dashed line displays the location of the corotation radius $r_{c}$. In the pressure and surface density panels, the nearly horizontal (saturated) structure is the planet's wake. The entropy and surface density panels show the propagation of perturbations sliding along the separatrices of the coorbital region, whereas the gas pressure is weakly perturbed.

As reported by Baruteau \& Masset (2008), these perturbations arise only in adiabatic disks, where a non-vanishing entropy gradient induces a discontinuity in the entropy and surface density fields at corotation, while the pressure field is continuous. In the linear limit, the perturbed fields (and their contribution to the torque) become singular, since the coorbital region becomes infinitely narrow. In our calculation, the unperturbed entropy profile is a decreasing function of the radius. The horseshoe dynamics therefore advects a negative entropy perturbation to the inner side of the coorbital region $\left(r<r_{c}\right)$, and a positive entropy perturbation to the outer side $\left(r>r_{c}\right)$. Since the pressure is only weakly perturbed, each entropy perturbation is related to a surface density perturbation of opposite sign and, in relative value, of same order of magnitude.

\section{Excess of corotation torque and entropy gradient}
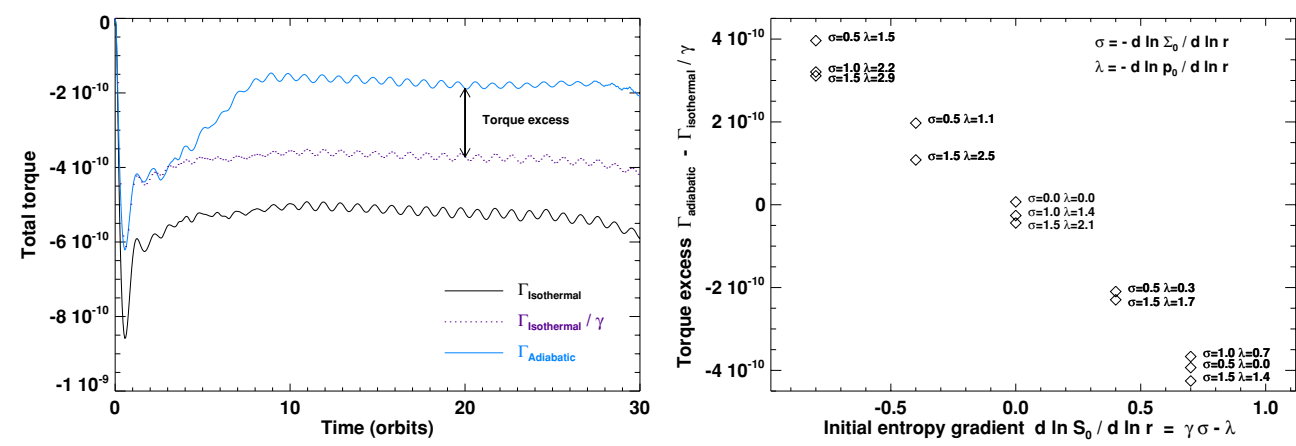

Figure 3. Left: torques obtained with the adiabatic and isothermal runs of section 2 . The dotted curve depicts the torque expected in the adiabatic situation when discarding the surface density perturbations advected by the horseshoe dynamics. Right: torque excess versus the unperturbed entropy gradient at corotation $\left(d \ln S_{0} / d \ln r\right)_{r_{c}}$, for different values of the unperturbed pressure and surface density gradients.

The torque variation with time of our adiabatic calculation is depicted in figure $3 \mathrm{a}$. The torque obtained with a locally isothermal equation of state is displayed for comparison. If there were no unperturbed entropy gradient, hence no entropy perturbations advected by the horseshoe dynamics, the adiabatic torque would be equal to the isothermal torque divided by the adiabatic index (dotted line, see section 2 ). The adiabatic run displays here a positive excess of corotation torque, which accounts for the perturbations advected by the horseshoe dynamics. The sign of this torque excess is related to the sign of the surface density perturbations inside the coorbital region, hence to the sign of the unperturbed entropy gradient at corotation.

As shown in figure $3 \mathrm{~b}$, the excess of corotation torque scales with the gradient of the unperturbed entropy at corotation $\left(d \ln S_{0} / d \ln r\right)_{r_{c}}$. A positive entropy gradient induces a negative torque excess, which accelerates inward migration. On the contrary, a negative entropy gradient yields a positive torque excess, which slows down type I migration. If 
the entropy gradient is sufficiently negative, the torque excess can be positive enough to reverse type I migration. However, the torque excess saturates as libration flattens out the entropy profile across the coorbital region. The long-term behavior of this process will depend on the irreversible processes at work in the disk (viscous heating, radiative cooling), which can maintain an unsaturated torque excess (forthcoming work).

\section{References}

Baruteau, C., \& Masset, F. 2008, ApJ, 672, 1054

Paardekooper, S.-J., \& Mellema, G. 2006, A\&BA, 459, L17

Ward, W. R. 1997, Icarus, 126, 261 